\title{
ON THE SIMILARITY OF CENTERED OPERATORS TO CONTRACTIONS
}

\author{
SRDJAN PETROVIĆ
}

(Communicated by Palle E. T. Jorgensen)

\begin{abstract}
In this paper we show that every power-bounded operator-weighted shift with commuting normal weights is similar to a contraction. As an application of this result, we reduce the problem of whether every centered powerbounded operator is similar to a contraction to the analogous question about quasi-invertible centered operators.
\end{abstract}

\section{INTRODUCTION}

Let $\mathscr{H}$ be a separable, infinite-dimensional, complex Hilbert space, and let $\mathscr{L}(\mathscr{H})$ denote the algebra of all bounded linear operators acting on $\mathscr{H}$. An operator $T$ in $\mathscr{L}(\mathscr{H})$ is said to be power-bounded if there exists an $M>0$ such that, for every positive integer $n,\left\|T^{n}\right\| \leq M$, and to be polynomially bounded if there exists a constant $K>0$ such that

$$
\|p(T)\| \leq K \sup \{|p(\xi)|:|\xi|=1\}
$$

for every polynomial $p$. For simplicity of reference, we shall denote the class of all power-bounded operators in $\mathscr{L}(\mathscr{H})$ by $P W(\mathscr{H})$ and the class of all polynomially-bounded operators in $\mathscr{L}(\mathscr{H})$ by $P B(\mathscr{H})$. An operator $T$ in $\mathscr{L}(\mathscr{H})$ is said to be similar to a contraction (notation: $T \in \mathscr{S} C(\mathscr{H})$ ) if there exists an invertible operator $X$ in $\mathscr{L}(\mathscr{H})$ such that $\left\|X T X^{-1}\right\| \leq 1$.

For a long time it was an open question whether $P B(\mathscr{H}) \subset \mathscr{S} C(\mathscr{H})$, but eventually Foguel [1] provided a counterexample, which Halmos simplified in [2]. Further analysis of this example inspired the latter to formulate explicitly in [3] the question whether

$$
P B(\mathscr{H}) \subset \mathscr{S} C(\mathscr{H}) \text {. }
$$

Of course, it is obvious from the definition and von Neumann's inequality for contraction operators [14] that $\mathscr{S} C(\mathscr{H}) \subset P B(\mathscr{H})$. Several authors have

Received by the editors September 1, 1992; presented at Great Plains Operator Theory Symposium meeting, Iowa City, Iowa, May 1992.

1991 Mathematics Subject Classification. Primary 47A65, 47B37.

This paper is part of the author's Ph.D. thesis, written at the University of Michigan under the direction of Professor Carl Pearcy. The author gratefully acknowledges partial support by Texas A\&M University during the period when this paper was being written. 
addressed the question whether (1) is valid (cf. [4-6, 10]), and we mention, in particular, some nice progress made by Paulsen [8], but, as of this writing, the question remains open. An affirmative answer to this problem would produce an instantaneous structure theory for the class $P B(\mathscr{H})$-namely, the one obtained by transporting the much studied structure theory for contraction operators (cf. [14]) via similarity transformations to the class $P B(\mathscr{H})$. Furthermore, an affirmative answer is known for some classes of operators including the compact operators [13] and the scalar-weighted shifts [11].

An operator $T \in \mathscr{L}(\mathscr{H})$ is said to be weakly centered if $T T^{*}$ commutes with $T^{*} T$. An operator $T \in \mathscr{L}(\mathscr{H})$ is said to be centered if the doubly infinite sequence

$$
\ldots, T^{2} T^{* 2}, T T^{*}, T^{*} T, T^{* 2} T^{2}, \ldots
$$

consists of mutually commuting operators. Centered operators have been studied in [7]. If we write the polar decomposition $T=U P$ of a centered operator, then it is possible (cf. [7]) to classify centered operators by considering the structure of the partial isometry $U$ in the decomposition above. Namely, a centered operator is of type $I$ if $U$ is a pure isometry, of type II if $U$ is a pure coisometry, of type III if $U$ is a direct sum of nilpotent power partial isometries, and of type IV if $U$ is unitary.

The pertinence of weakly centered and centered operators to the question of whether (1) holds arises from the fact that in [10] the author reduced the question (1) to the question of whether every weakly centered, polynomiallybounded operator is similar to a contraction. Since centered operators are the best known examples of weakly centered operators, this naturally led to the question of whether every centered polynomially-bounded operator is similar to a contraction. It is the purpose of this paper to show that every powerbounded centered operator of type I, II, or III is similar to a contraction and thus that every centered operator in $P W$ belongs to $\mathscr{S C}$ if and only if every such operator of type IV does.

Before stating the main results of this paper we briefly mention some notation and terminology. As usual, $\mathbb{N}$ is the set of positive integers, $\mathbb{N}_{0}$ is the set of nonnegative integers, and $\mathbb{Z}$ denotes the set of all integers. If $\left\{A_{n}\right\}_{n \in \mathbb{Z}}$ is a sequence of operators in $\mathscr{L}(\mathscr{H})$, we denote by $\operatorname{diag}\left(\left\{A_{n}\right\}_{n=-\infty}^{\infty}\right)$ the direct sum $\bigoplus_{n=-\infty}^{\infty} A_{n}$ acting on $\mathscr{H}(\mathbb{Z})$, the direct sum of $\aleph_{0}$ copies of $\mathscr{H}$ indexed by $\mathbb{Z}$, in the obvious fashion. As usual $C(X)$ is the algebra of all continuous complex-valued functions on $X$. A topological space $X$ is said to be extremally disconnected if $X$ has a topological base consisting of compact open sets and the closure of every open set is open.

Theorem 1.1. Every centered operator of type I, II, or III, as well as any direct sum of such operators, is similar to a contraction if and only if it is power-bounded.

The proof of Theorem 1.1 depends on an analysis of several cases. By a theorem of Morrel and Muhly [7], every centered operator of type I is unitarily equivalent to a forward, unilateral, operator-weighted shift whose weights are mutually commuting, Hermitian, quasi-invertible operators. Theorem 1.1 asserts that such an operator is similar to a contraction if and only if it is power-bounded. However, more is true. 
Theorem 1.2. If $T \in \mathscr{L}(\mathscr{H})$ is a (forward, backward, or bilateral) operatorweighted shift with commuting normal operators as weights, then $T \in \mathscr{S} C(\mathscr{H})$ if and only if $T \in P W(\mathscr{H})$.

\section{Proof of Theorem 1.2}

Proof. Since the inclusion $\mathscr{S} C(\mathscr{H}) \subset P B(\mathscr{H})$ is well known, we concentrate on the opposite inclusion. We will give the proof in the case when $T$ is a bilateral (forward) operator-weighted shift. The case when $T$ is a forward, unilateral, operator-weighted shift can be expounded in a similar way, and the case when $T$ is a backward shift (unilateral or bilateral) follows from the facts that a backward shift is the adjoint of a forward one and that the classes $P W$ and $\mathscr{S} C$ are selfadjoint.

Given a bilateral (forward) operator-weighted shift $T$ with weight sequence $\left\{A_{i}\right\}_{i \in \mathbb{Z}}$ consisting of mutually commuting normal operators, let $\mathscr{A}$ be the (abelian) von Neumann algebra generated by the sequence $\left\{A_{i}\right\}_{i \in \mathbb{Z}}$. Then one knows (see [12]) that there exists an extremally disconnected, compact Hausdorff space $X$ and an isometric *-isomorphism $\rho$ of $C(X)$ onto $\mathscr{A}$. Let $f_{n}=\rho^{-1}\left(A_{n}\right), n \in \mathbb{Z}$. Also, there exists a decomposition $\mathscr{H}=\sum \bigoplus_{n=-\infty}^{\infty} \mathscr{H}_{n}$ of the Hilbert space $\mathscr{H}$ such that $A_{i}\left(\mathscr{H}_{i}\right) \subset \mathscr{H}_{i+1}$. We will construct a bounded invertible operator $S$ of the form

$$
\operatorname{diag}\left(\left\{\rho\left(g_{n}\right)\right\}_{n=-\infty}^{\infty}\right),
$$

relative to the same decomposition of $\mathscr{H}$ (where the sequence $\left\{g_{n}\right\} \subset C(X)$ is to be constructed), such that $S^{-1} T S$ is a contraction.

First we define functions $\left\{\tilde{f}_{n}\right\}_{n \in \mathbb{Z}}$ in $C(X)$ inductively. Let $x \in X$. We define

$$
\tilde{f}_{0}(x)=\max \left\{\left|f_{0}(x)\right|, 1\right\}
$$

and notice that $\tilde{f}_{0}(x) \neq 0$ for $x \in X$. If $n \in \mathbb{N}$ and $\tilde{f}_{0}(x), \tilde{f}_{1}(x), \ldots, \tilde{f}_{n-1}(x)$ are defined and invertible, then for each $x \in X$ we set

$$
\begin{aligned}
p_{i, j}(x) & = \begin{cases}\left|\prod_{k=i}^{j} f_{k}(x)\right|, & i \leq j, \\
1, & \text { otherwise } ;\end{cases} \\
\tilde{p}_{i, j}(x) & = \begin{cases}\prod_{k=i}^{j} \tilde{f}_{k}(x), & i \leq j, \\
1, & \text { otherwise }\end{cases} \\
q_{n}(x) & =\min \left\{1, \min _{0 \leq i \leq n-1} \frac{1}{\tilde{p}_{i, n-1}(x)}\right\} ; \\
r_{n}(x) & =\min \left\{1, \min _{n+1 \leq j \leq-1} \frac{1}{\tilde{p}_{n+1, j}(x)}\right\} ;
\end{aligned}
$$

and

Next, we define

$$
\tilde{f}_{n}(x)=\max \left\{\left|f_{n}(x)\right|, q_{n}(x)\right\} \text {. }
$$

$$
\tilde{f}_{-1}(x)=\max \left\{\left|f_{-1}(x)\right|, 1\right\},
$$

and, as before, if $n$ is a negative integer different from -1 and $\tilde{f}_{-1}(x), \tilde{f}_{-2}(x)$, $\ldots, \tilde{f}_{n+1}(x)$ are defined and invertible, then for each $x \in X$ we set

$$
\tilde{f}_{n}(x)=\max \left\{\left|f_{n}(x)\right|, r_{n}(x)\right\} .
$$


We see that each $\tilde{f}_{n}$ is a positive invertible function in $C(X)$ and, moreover, that the bilateral operator-weighted shift $\tilde{T}$ with weight sequence $\left\{\rho\left(\tilde{f}_{n}\right)\right\}_{n \in \mathbb{Z}}$ is power-bounded. Indeed, we shall verify that for all $m, n \in \mathbb{Z}$ with $m \leq n$

$$
\left\|\prod_{k=m}^{n} \rho\left(\tilde{f}_{k}\right)\right\| \leq M^{2}
$$

Let $J$ be the set of all integers $k$ that satisfy $m \leq k \leq n$, and let $J_{+}$(respectively, $J_{-}$) be the subsets of $J$ consisiting of its nonnegative (respectively, negative) elements. The expression on the left-hand side of the last inequality equals

$$
\begin{aligned}
\left\|\prod_{k \in J_{-}} \rho\left(\tilde{f}_{k}\right) \prod_{k \in J_{+}} \rho\left(\tilde{f}_{k}\right)\right\| & =\left\|\rho\left(\prod_{k \in J_{-}} \tilde{f}_{k} \prod_{k \in J_{+}} \tilde{f}_{k}\right)\right\| \|_{C(X)} \\
& \leq \sup _{x \in X}\left|\prod_{k \in J_{-}} \tilde{f}_{k}(x)\right| \sup _{x \in X}\left|\prod_{k \in J_{+}} \tilde{f}_{k}(x)\right| .
\end{aligned}
$$

Now we show that for each $x$ in $X$ both

$$
\left|\prod_{k \in J_{-}} \tilde{f}_{k}(x)\right| \leq M
$$

and

$$
\left|\prod_{k \in J_{+}} \tilde{f}_{k}(x)\right| \leq M
$$

In order to do that, we will show that for each $x$ in $X$

$$
\tilde{p}_{m, n}(x) \leq \max \left\{1, \max _{m \leq j \leq n} p_{m, j}(x)\right\}
$$

for any $m \leq n<0$ and that

$$
\tilde{p}_{m, n}(x) \leq \max \left\{1, \max _{m \leq i \leq n} p_{i, n}(x)\right\}
$$

for any $0 \leq m \leq n$.

Let $x$ in $X$ and $n<0$ be fixed. We will prove $\left(3^{\prime}\right)$ by induction on $m$. If $m=n$, we need to show that $\tilde{f}_{n}(x) \leq \max \left\{1,\left|f_{n}(x)\right|\right\}$. If $\left|f_{n}(x)\right| \leq r_{n}(x)$, then $\tilde{f}_{n}(x) \leq 1$. If $\left|f_{n}(x)\right|>r_{n}(x)$, then $\tilde{f}_{n}(x)=\left|f_{n}(x)\right|$. Let $\left(3^{\prime}\right)$ be true for some $m \leq n$. We will prove that it will still be valid with $m-1$ instead of $m$. If $\left|f_{m-1}(x)\right| \leq r_{m-1}(x)$, then

$$
\tilde{f}_{n}(x)=r_{m-1}(x) \leq \min _{m \leq j \leq-1} \frac{1}{\tilde{p}_{m, j}(x)} \leq \frac{1}{\tilde{p}_{m, n}(x)}
$$

so that $\tilde{p}_{m-1, n}(x)=\tilde{f}_{m-1}(x) \tilde{p}_{m, n}(x) \leq 1$. If $\left|f_{m-1}(x)\right|>r_{m-1}(x)$, then 


$$
\begin{aligned}
& \tilde{f}_{m-1}(x)=\left|f_{m-1}(x)\right| \text { so that } \\
& \begin{aligned}
\tilde{p}_{m-1, n}(x) & =\left|f_{m-1}(x)\right| \tilde{p}_{m, n}(x) \leq\left|f_{m-1}(x)\right| \max \left\{1, \max _{m \leq j \leq n} p_{m, j}(x)\right\} \\
& =\max \left\{\left|f_{m-1}(x)\right|, \max _{m \leq j \leq n}\left|f_{m-1}(x)\right| p_{m, j}(x)\right\} \\
& =\max \left\{p_{m-1, m-1}(x), \max _{m \leq j \leq n} p_{m-1, j}(x)\right\} \\
& =\max _{m-1 \leq j \leq n} p_{m-1, j}(x) .
\end{aligned}
\end{aligned}
$$

Inequality $\left(4^{\prime}\right)$ can be proved in an analogous way, namely, by induction on $n$. We omit the details. The power-boundedness of $T$ implies that the right-hand sides of $\left(3^{\prime}\right)$ and $\left(4^{\prime}\right)$ are bounded by $M$, which shows that $(3)$ and (4) are true. We conclude that $\tilde{T}$ is in the class $P W$.

Now we define the functions $\left\{g_{n}\right\}$ for each $n \in \mathbb{Z}$ :

$$
g_{n}(x)= \begin{cases}\frac{\tilde{p}_{0, n-1}(x)}{\min _{0 \leq k \leq n} \tilde{p}_{0, k-1}(x)} & \text { if } n \in \mathbb{N}_{0}, \\ \frac{\min _{n \leq k \leq 0} \tilde{p}_{k,-1}(x)}{\tilde{p}_{n,-1}(x)} & \text { if } n=-1,-2, \ldots\end{cases}
$$

It is obvious that each $g_{n}$ is in $C(X)$. Therefore, for each $n$ in $\mathbb{Z}$, the operator $\rho\left(g_{n}\right)$ is well defined. Furthermore, for $n \in \mathbb{N}$,

$$
\left\|\rho\left(g_{n}\right)\right\|=\left\|g_{n}\right\|=\left\|\frac{\tilde{p}_{0, n-1}(x)}{\min _{0 \leq k \leq n} \tilde{p}_{0, k-1}(x)}\right\|=\left\|\frac{\tilde{p}_{0, n-1}(x)}{\tilde{p}_{0, k-1}(x)}\right\|
$$

for some $k$ satisfying $0 \leq k \leq n$, and therefore

$$
\left\|\rho\left(g_{n}\right)\right\|=\left\|\tilde{p}_{k, n-1}(x)\right\| \leq M .
$$

On the other hand, for $n=-1,-2, \ldots$,

$$
\left\|\rho\left(g_{n}\right)\right\|=\left\|g_{n}\right\|=\left\|\frac{\min _{n \leq k \leq 0} \tilde{p}_{k,-1}(x)}{\tilde{p}_{n,-1}(x)}\right\| \leq 1,
$$

and since, obviously, $g_{0}(x)=1$, it follows that the operator $S$ defined in (2) is a bounded linear operator.

Moreover, it is invertible. To see that, let a sequence $\left\{h_{n}\right\}_{n \in \mathbb{Z}} \subset C(X)$ be defined as

$$
h_{n}(x)=\frac{1}{g_{n}(x)}, \quad x \in X
$$

Since $g_{n}(x) \neq 0$ for every $n \in \mathbb{Z}$ and for all $x \in X$, each function $h_{n}$ is in $C(X)$, and hence the operators $\rho\left(h_{n}\right)$ are well defined, bounded, linear operators.

Next, we notice that for $n \in \mathbb{N}$

$$
\left\|h_{n}\right\|=\left\|\frac{1}{g_{n}}\right\|=\left\|\frac{\min _{0 \leq k \leq n} \tilde{p}_{0, k-1}(x)}{\tilde{p}_{0, n-1}(x)}\right\| \leq 1,
$$

and that for $n=-1,-2, \ldots$

$$
\left\|h_{n}\right\|=\left\|\frac{1}{g_{n}}\right\|=\left\|\frac{\tilde{p}_{n,-1}(x)}{\min _{n \leq k \leq 0} \tilde{p}_{k,-1}(x)}\right\|=\left\|\frac{\tilde{p}_{n,-1}(x)}{\tilde{p}_{k,-1}(x)}\right\|
$$


for some $k$ satisfying $n \leq k \leq 0$, and therefore

$$
\left\|h_{n}\right\|=\left\|\tilde{p}_{n, k-1}(x)\right\| \leq M .
$$

Together with the fact that $h_{0}(x)=1$, this shows that the operator

$$
R=\operatorname{diag}\left(\left\{\rho\left(h_{n}\right)\right\}_{n=-\infty}^{\infty}\right)
$$

is a bounded linear operator. Since $g_{n} h_{n} \equiv 1$ for all $n, S R=R S=I$, which shows that $S$ is an invertible operator whose inverse is $R$.

Finally, we show that $S^{-1} T S$ is a contraction. Since $S^{-1} T S$ is a bilateral (forward) operator-weighted shift with weight sequence $\left\{\rho\left(h_{n+1} f_{n} g_{n}\right)\right\}_{n \in \mathbb{Z}}$, it suffices to prove that for all $n \in \mathbb{Z}$ the operators $\rho\left(h_{n+1} f_{n} g_{n}\right)$ are contractions. We notice that $\left|f_{n}(x)\right| \leq \tilde{f}_{n}(x)$, so it suffices to show that

$$
\left\|h_{n+1} \tilde{f}_{n} g_{n}\right\| \leq 1, \quad n \in \mathbb{Z} .
$$

Since

$$
\left\|h_{n+1} \tilde{f}_{n} g_{n}\right\|=\sup _{x \in X}\left|h_{n+1}(x) \tilde{f}_{n}(x) g_{n}(x)\right|=\left|\frac{1}{g_{n+1}(x)} \tilde{f}_{n}(x) g_{n}(x)\right|,
$$

it suffices to show that for any $x$ in $X$

$$
\frac{1}{g_{n+1}(x)} \tilde{f}_{n}(x) g_{n}(x) \leq 1 \text {. }
$$

First we consider the case $n \in \mathbb{N}$. We fix $x \in X$. Then the left-hand side of (6) becomes

$$
\begin{aligned}
& \frac{\min _{0 \leq k \leq n+1} \tilde{p}_{0, k-1}(x)}{\tilde{p}_{0, n}(x)} \tilde{f}_{n}(x) \frac{p_{0, n-1}(x)}{\min _{0 \leq k \leq n} \tilde{p}_{0, k-1}(x)} \\
& \quad=\frac{\min _{0 \leq k \leq n+1} \tilde{p}_{0, k-1}(x)}{\min _{0 \leq k \leq n} \tilde{p}_{0, k-1}(x)} \leq 1 .
\end{aligned}
$$

In the case $n=0$, taking into account that $g_{0}(x)=1$, the left-hand side of (6) reduces to

$$
\begin{aligned}
\frac{1}{g_{1}(x)} \tilde{f}_{0}(x) & =\frac{\min _{0 \leq k \leq 1} \tilde{p}_{0, k-1}(x)}{\tilde{p}_{0,0}(x)} \tilde{f}_{0}(x) \\
& =\min \left\{\tilde{p}_{0,-1}(x), \tilde{p}_{0,0}(x)\right\} \leq \tilde{p}_{0,-1}(x)=1 .
\end{aligned}
$$

Similarly, if $n=-1$, we obtain

$$
\begin{aligned}
\tilde{f}_{-1}(x) g_{-1}(x) & =\tilde{f}_{-1}(x) \frac{\min _{-1 \leq k \leq 0} \tilde{p}_{k,-1}(x)}{\tilde{p}_{-1,-1}(x)} \\
& =\min \left\{\tilde{p}_{-1,-1}(x), \tilde{p}_{0,-1}(x)\right\} \leq \tilde{p}_{0,-1}(x)=1 .
\end{aligned}
$$

Finally, if $n=-2,-3, \ldots$,

$$
\frac{\tilde{p}_{n+1,-1}(x)}{\min _{n+1 \leq k \leq 0} \tilde{p}_{k,-1}(x)} \tilde{f}_{n}(x) \frac{\min _{n \leq k \leq 0} \tilde{p}_{k,-1}(x)}{\tilde{p}_{n,-1}(x)}=\frac{\min _{n \leq k \leq 0} \tilde{p}_{k,-1}(x)}{\min _{n+1 \leq k \leq 0} \tilde{p}_{k,-1}(x)} \leq 1 .
$$

Thus, $\left\|S^{-1} T S\right\| \leq 1$, and the theorem is proved. 
Remark 2.1. It is easy to see that the similarity $S$ constructed in the proof of the previous theorem satisfies $\|S\| \leq M$ and $\left\|S^{-1}\right\| \leq M$, where $M$ is the power bound of $T$.

\section{Continuation of the PROOF OF Theorem 1.1}

Proof. Let $T$ be a centered, power-bounded operator in $\mathscr{L}(\mathscr{H})$ with power bound $M$. One knows (cf. [7]) that if $T$ is of type I (respectively, type II), then $T$ is unitarily equivalent to a forward (respectively, backward), unilateral operator-weighted shift with weights which are mutually commuting, hermitian, quasi-invertible operators. Thus, in both cases, the assertion is exactly Theorem 1.2 .

If $T$ is of type III, then $T$ can be written as a direct sum $T=\bigoplus_{n=1}^{\infty} T_{n}$, acting on $\bigoplus_{n=1}^{\infty} \mathscr{H}_{n}$, where each $T_{n}$ is acting on the space (0) or it is unitarily equivalent to the operator matrix

$$
\left(\begin{array}{ccccc}
0 & & & & \\
A_{1, n} & 0 & & & \\
& A_{2, n} & 0 & & \\
& & \ddots & \ddots & \\
& & & A_{n-1, n} & 0
\end{array}\right)
$$

and $A_{1, n}, A_{2, n}, \ldots, A_{n-1, n}$ are mutually commuting, hermitian, quasiinvertible operators. In that case, there exist Hilbert spaces $\mathscr{K}_{n} \supset \mathscr{H}_{n}$ and operators $\hat{T}_{n} \in \mathscr{L}\left(\mathscr{K}_{n}\right)$ such that each $\hat{T}_{n}$ is an operator-weighted shift with weights $A_{1, n}, A_{2, n}, \ldots, A_{n-1, n}, 0,0, \ldots$; each $\mathscr{H}_{n}$ is invariant for $\hat{T}_{n}$; and $\hat{T}_{n} \mid \mathscr{H}_{n}=T_{n}$. It is not hard to see that, for every $n \in \mathbb{N}, \hat{T}_{n}$ satisfies the hypotheses of Theorem 1.2, and since $\hat{T}_{n}$ is power-bounded, there exists a family of invertible operators $\left\{\hat{S}_{n}\right\}$ such that $\left\|\hat{S}_{n}^{-1} \hat{T}_{n} \hat{S}_{n}\right\| \leq 1$. This implies that there exists another family of operators $\left\{S_{n}\right\}$ such that $\left\|S_{n}{ }^{-1} T_{n} S_{n}\right\| \leq 1$, and in addition, by Remark 2.1, $\left\|S_{n}\right\| \leq\left\|\hat{S}_{n}\right\| \leq M$ and $\left\|S_{n}{ }^{-1}\right\| \leq\left\|\hat{S}_{n}^{-1}\right\| \leq M$. Then it is easy to see that the operator $S=\bigoplus_{n=1}^{\infty} S_{n}$ is a bounded invertible operator on $\bigoplus_{n=1}^{\infty} \mathscr{H}_{n}$ and that $S$ satisfies $\left\|S^{-1} T S\right\| \leq 1$ as well as $\|S\|,\left\|S^{-1}\right\| \leq M$.

Thus, for each of the types I, II, or III the similarity is bounded (uniformly) from above and from below, which shows that a direct sum of such operators is similar to a contraction. This completes the proof of Theorem 1.1.

\section{Problems}

Since every centered operator is an arbitrary direct sum of operators of types I, II, III, and IV, it remains to investigate the case when a centered powerbounded operator is of type IV. The structure of centered operators of type IV is set forth in Theorem 4.2, which is taken from [7].

If $\mathscr{H}$ is a Hilbert space and $T \in \mathscr{L}(\mathscr{H})$ is a quasi-invertible operator with polar decomposition $T=U P$, then let $\theta_{T}$ denote the $C^{*}$-algebra generated by the operators $\left\{\left(U^{*}\right)^{n} P U^{n}\right\}_{n=-\infty}^{+\infty}$ together with the identity operator. If $X$ is a compact Hausdorff space, $\mu$ is a positive, regular, Borel measure on $X$, and $\mathscr{G}$ is a Hilbert space, then $L_{\mathscr{G}}^{2}(\mu)$ will denote the Hilbert space of all measurable $\mathscr{G}$-valued functions on $X$ which are square-integrable with respect to $\mu$. If $\varphi \in L^{\infty}(\mu)$, then $M_{\varphi}$ will denote the multiplication operator on 
$L_{\mathscr{G}}^{2}(\mu)$ defined by $\left(M_{\varphi} f\right)(x)=\varphi(x) f(x)$ for all $f \in L_{\mathscr{G}}^{2}(\mu)$. An operator $A$ on $L_{\mathscr{G}}^{2}(\mu)$ is said to be decomposable if there is a bounded, measurable, $\mathscr{L}(\mathscr{G})$ valued function $A(x)$ on $X$ such that $(A f)(x)=A(x) f(x)$ for every $x \in X$ and every $f \in L_{\mathscr{S}}^{2}(\mu)$.

Definition 4.1. A type IV centered operator $T$ will be called a type IV $_{n}$ centered operator, $n=1,2, \ldots, \infty$, provided that $\theta_{T}^{\prime \prime}$, the abelian von Neumann algebra generated by $\theta_{T}$, has uniform multiplicity $n$.

Finally, we come to a model for type IV centered operators.

Theorem 4.2. Let $\mathscr{H}$ be a Hilbert space, and let $T \in \mathscr{L}(\mathscr{H})$ be a type IV centered operator. Then there exists a decomposition $\mathscr{H}=\bigoplus_{i \in I} \mathscr{H} i$ of $\mathscr{H}$ consisting of reducing subspaces $\mathscr{H}_{i}$ for $T$ such that, relative to this decomposition, $T \mid \mathscr{H}_{i}$ is a centered operator of type $\mathrm{IV}_{n_{i}}$. Furthermore, if $T$ is a type $\mathrm{IV}_{n}$ centered operator with polar decomposition $T=U P, X$ is the maximal ideal space of the commutative $C^{*}$-algebra $\theta_{T}$, and $\Gamma$ denotes the Gelfand transform of $\theta_{T}$ onto $C(X)$, then there exists

(1) a homeomorphism $\tau$ of $X$ onto $X$,

(2) a finite measure $\mu$ on $X$ which is quasi-invariant under $\tau$,

(3) an $n$-dimensional Hilbert space $\mathscr{G}$,

(4) a decomposable unitary operator $\Theta$ on $L_{\mathscr{G}}^{2}(\mu)$, and

(5) a Hilbert space isomorphism $W$ from $\mathscr{H}$ onto $L_{\mathscr{G}}^{2}(\mu)$

such that

$$
W T W^{-1}=\Theta S M_{\Gamma(P)},
$$

where $S$ is the unitary operator defined on $L_{\mathscr{G}}^{2}(\mu)$ by the formula

$$
(S f)(x)=f(\tau(x))(d(\mu \circ \tau) / d \mu)^{1 / 2}(x)
$$

for all $f \in L_{\mathscr{G}}^{2}(\mu)$.

As is easily seen, this model is much different from the models for centered operators of types I, II, and III, and it seems that it is necessary to apply somewhat different techniques in order to obtain an answer to the similarity problem.

As mentioned above it was shown in [10] that every polynomially bounded operator has a dilation that is weakly centered.

Problem 4.3. Is every weakly centered polynomially-bounded operator similar to a contraction?

Problem 4.4. Does every polynomially-bounded operator have a dilation that is centered?

Recall that an operator $T$ in $\mathscr{L}(\mathscr{H})$ is said to be $n$-normal if it is unitarily equivalent to an $n \times n$ matrix with mutually commuting, normal entries. Obviously, each centered operator of type III $_{n}$ is an $n$-normal operator.

Problem 4.5. Is every $n$-normal, polynomially-bounded operator similar to a contraction?

Remark 4.6. Very recently the author, in collaboration with C. Pearcy and $\mathrm{V}$. Paulsen, proved that every centered operator of type IV is similar to a contraction if and only if it is power bounded. This result will appear in [9]. 


\section{REFERENCES}

1. S. R. Foguel, A counterexample to a problem of Sz.-Nagy, Proc. Amer. Math. Soc. 15 (1964), 788-790.

2. P. R. Halmos, On Foguel's answer to Nagy's question, Proc. Amer. Math. Soc. 15 (1964), 791-793.

3. __ Ten problems in Hilbert space, Bull. Amer. Math. Soc. 76 (1970), 887-933.

4. J. A. R. Holbrook, Spectral dilations and polynomially bounded operators, Indiana Univ. Math. J. 20 (1971), 1027-1034.

5. A. Lebow, On von Neumann's theory of spectral sets, J. Math. Anal. Appl. 7 (1963), 64-90.

6. W. Mlak, Algebraic polynomially bounded operators, Ann. Polon. Math. 29 (1974), 133-139.

7. B. Morrel and P. Muhly, Centered operators, Studia Math. 51 (1974), 251-263.

8. V. I. Paulsen, Every completely polynomially bounded operator is similar to a contraction, J. Funct. Anal. 55 (1984), 1-17.

9. V. I. Paulsen, C. M. Pearcy, and S. Petrović, On centered and weakly centered operators, J. Funct Anal. (submitted).

10. S. Petrović, A dilation theory for polynomially bounded operators, J. Funct. Anal. 108 (1992), 458-469.

11. A. L. Shields, Weighted shift operators and analytic function theory, Topics in Operator Theory, Math. Monographs Surveys, vol. 13, Amer. Math. Soc., Providence, RI, 1974, pp. $49-128$.

12. M. H. Stone, Boundedness properties in function lattices, Canad. J. Math 1 (1949), 176-186.

13. B. Sz.-Nagy, Completely continuous operators with uniformly bounded iterates, Magyar Tud. Akad. Mat. Kutató Int. Köze 4 (1959), 89-93.

14. B. Sz.-Nagy and C. Foias, Harmonic analysis of operators on Hilbert space, American Elsevier, New York, 1970.

Department of Mathematics, Texas A\&M University, College Station, Texas 77843

Current address: Department of Mathematics, Indiana University, Bloomington, Indiana 47405

E-mail address: petrovic@iu-math.math.indiana.edu 